LAWRENCE LIVERMORE N A TION AL LABORATORY
Validating the Predicted Lateral Straggling of MeV-Energy Proton Beams

G. Bench, T. Felter, A. Antolak

November 20, 2003 
This document was prepared as an account of work sponsored by an agency of the United States Government. Neither the United States Government nor the University of California nor any of their employees, makes any warranty, express or implied, or assumes any legal liability or responsibility for the accuracy, completeness, or usefulness of any information, apparatus, product, or process disclosed, or represents that its use would not infringe privately owned rights. Reference herein to any specific commercial product, process, or service by trade name, trademark, manufacturer, or otherwise, does not necessarily constitute or imply its endorsement, recommendation, or favoring by the United States Government or the University of California. The views and opinions of authors expressed herein do not necessarily state or reflect those of the United States Government or the University of California, and shall not be used for advertising or product endorsement purposes.

This work was performed under the auspices of the U.S. Department of Energy by University of California, Lawrence Livermore National Laboratory under Contract W-7405-Eng-48. 


\title{
Validating the Predicted Lateral Straggling of MeV-Energy Proton Beams
}

\author{
Graham Bench and Thomas Felter \\ Lawrence Livermore National Laboratory \\ Livermore, CA 94551 \\ Arlyn Antolak \\ Analytical Materials Science Department \\ Sandia National Laboratories \\ P.O. Box 969 \\ Livermore, CA 94551-9403
}

\begin{abstract}
Proton imaging is a potential nondestructive method for characterizing NIF (National Ignition Facility) targets in two- and three-dimensions with micron-scale spatial resolution. The main limitation for high resolution imaging with proton beams, especially for thick samples, is the positional blurring of the proton beam, known as "lateral straggling". Accurate prediction of the amount of lateral straggling and, consequently, the achievable spatial resolution in pertinent NIF target material combinations and geometries requires validated proton transport models. We present results of Monte Carlo simulations of $\mathrm{MeV}$-energy proton transport through thin $(\sim 1$ micron thick) metal foils. The calculated residual proton distributions are compared to recent lateral straggling measurements obtained at the LLNL 4-MV Pelletron accelerator.
\end{abstract}

\section{Acknowledgements}

This work was supported by the U.S. Department of Energy under Sandia National Laboratories Contract DE-AC04-94AL85000 and Lawrence Livermore National Laboratory contract W-7405 ENG-48. The authors would like to thank H. Martz and W. Hsing at LLNL for suggesting this work in order to assess the accuracy of estimates of the achievable spatial resolution in proton imaging. 


\section{Contents}

I. Introduction.................................................

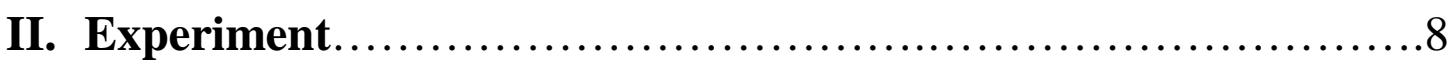

III. Ion Transport Modeling. ...................................

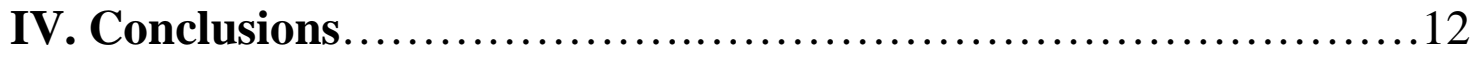

V. References.................................................. 13

\section{Figures}

1. Measured and calculated lateral straggling distributions for MeV-energy protons incident on an aluminum foil.............................. 14

2. Measured and calculated lateral straggling distributions for MeV-energy

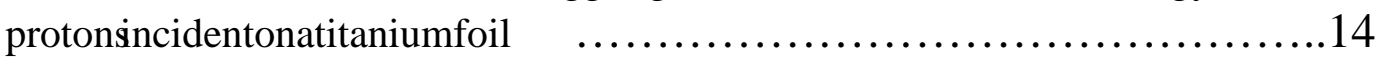

3. Measured and calculated lateral straggling distributions for $M e V$-energy protons incident on a nickel foil................................ 15

4. Measured and calculated lateral straggling distributions for MeV-energy protons incident on a silver foil................................. 15

5. Measured and calculated lateral straggling distributions for $M e V$-energy protons incident on a tungsten foil................................. 16

6. Measured and calculated lateral straggling distributions for MeV-energy protons incident on a gold foil .................................. 16

7. Relative error of TRIM-calculated lateral broadening HWHM values for 2.5 - and $3.5-\mathrm{MeV}$ protons on thin metal foils...................... 17

\section{Tables}

1. HWHM (Half Width at Half Maximum) values of the measured and predicted lateral straggling distributions from thin metal foils................10

2. RMS (Root-Mean-Square) scattering angles for $2.5-\mathrm{MeV}$ and $3.5-\mathrm{MeV}$ protons on thin metal foils 


\section{Validating the Predicted Lateral Straggling of MeV-Energy Proton Beams}

\section{Introduction}

Focused proton beam imaging is a potential nondestructive method for characterizing mesoscale-sized objects, such as NIF (National Ignition Facility) targets, with micron-scale spatial resolution. Protons in the few-to$100-\mathrm{MeV}$ range lose energy continuously and predictably to matter, predominantly by electron-hole pair production. Therefore, measuring the energy loss of a proton beam that has traveled through a target provides information about the line integrated electron density in a single measurement. Repeating this measurement across the sample leads to a twodimensional (areal) density mapping of the sample known as a STIM (scanning transmission ion microscopy) image, which is analogous to an $\mathrm{x}$ ray radiograph. Further, by acquiring multiple STIM images as the sample is rotated in small angular increments, a full volumetric rendering of the sample (density) can be reconstructed and visualized. This latter imaging technique, called IMT (ion micro-tomography), is analogous to $\mathrm{x}$-ray computed tomography (CT). The power of sufficiently energetic proton beams to penetrate thick targets, together with the potential for highdynamic-range measurements enabled by the roughly linear energy loss mechanism in the material, makes proton beam imaging especially useful in characterizing objects where large variations in atomic number or low total density limit the applicability of x-ray CT analysis. ${ }^{1-3}$

Although the interaction of charged particle beams with matter is well understood, high-resolution imaging with focused proton beams is a more recent development. The main limitation for high spatial resolution with proton beams is the positional blurring of the proton beam, known as "lateral straggling". This blurring is caused by the beam's strong interactions with the electronic charge distribution of the material through which it travels. Multiple scattering of individual ions results in small, cumulative directional changes that ultimately cause spatial broadening of the original focused beam. Even with incident micron-sized beams, lateral straggling can limit the exit beam profile to several tens of microns in some cases. In addition, the protons acquire a distribution of exit energies along their direction of travel, depending on the collision statistics of their trajectories through the target. This latter effect is called "energy straggling." Energy straggling 
determines the accuracy with which the energy loss measurement can be performed and, subsequently, the density contrast of the image. ${ }^{4-6}$

It was not until the mid-1990's that the problem of image degradation caused by straggling began to be addressed through image reconstruction algorithms that corrected for the effect of beam spatial broadening., ${ }^{7,8}$ Lateral straggling will always occur at some level in proton imaging characterization, but its affect can be mitigated using a correction algorithm as described in Ref. 8. Following that approach, a first order reconstruction is made of the object's geometry using the acquired projection data, $P_{\text {meas }}$. This first order rendering is then used as a basis for generating two new sets of projection data, one set corresponding to the case that includes beam spatial broadening effects, $P_{\text {stragg, }}$, and the other without, $P_{\text {nostragg. }}$ The correction algorithm generates a final projection data set by iteratively adjusting the measured and simulated projection data sets according to

$$
P_{\text {corr }}=P_{\text {meas }}-P_{\text {stragg }}+P_{\text {nostragg }} .
$$

The main difficulty lies with the evaluation of $P_{\text {stragg }}$ which requires a voxelby-voxel $^{*}$ calculation of the root-mean-square scattering angle of the ion beam as it traverses each voxel. In Ref. 8, an approximate analytical expression was used for the scattering angle, applicable to samples consisting of only a single element. ${ }^{9}$ On the other hand, complex targets, e.g., NIF targets, necessitate developing an improved correction algorithm that directly incorporates accurate scattering angle information for determining $P_{\text {stragg. }}$. Such scattering information can be derived from Monte Carlo ion transport calculations provided the predicted lateral straggling distributions are validated to experimental distributions.

In this report, we present comparisons of predicted proton lateral straggling distributions to recently measured ones obtained using incident 2.5- to 3.5$\mathrm{MeV}$ proton beams on thin metal calibration foils. The next section describes the proton beam experimental arrangement and data collection process; the following sections show graphical plots of the computed and measured distributions, and a summary of the results.

\footnotetext{
*A voxel is the smallest distinguishable (generally) cube-shaped part of a three-dimensional space. A particular voxel, or "volume element", is usually identified by the $\mathrm{x}, \mathrm{y}$, and $\mathrm{z}$ coordinates of its center.
} 


\section{Experiment}

Measurements of the transmitted proton beam intensity as a function of angle were obtained on thin metal foil samples at the LLNL (Lawrence Livermore National Laboratory) 4 MV Pelletron accelerator. The foils used in the beam broadening experiments were $\mathrm{Al}, \mathrm{Ti}, \mathrm{Ni}, \mathrm{Ag}, \mathrm{W}$, and $\mathrm{Au}$ having a vendor-supplied nominal thickness of 1 micron (except for nickel and tungsten having nominal thicknesses of $0.5 \mu \mathrm{m}$ and $3 \mu \mathrm{m}$, respectively). We obtained an independent gravimetric determination of each film's thickness (see Table 1) so that more accurate values would be used in the simulations. All films except nickel had gravimetric thicknesses within $20 \%$ of their reported nominal thickness. Each sample was mounted in a holder that was placed in the analysis chamber of the beamline. For $2.5-$ to $3.5-\mathrm{MeV}$ protons, the backscattering cross-sections for some of the low $\mathrm{Z}$ targets display significant departures from Rutherford scattering cross-sections, so backscattering determinations of specimen thickness were not performed.

A silicon surface barrier detector mounted on an x-y stage was collimated with a $1 \mathrm{~mm}$ diameter aperture and was positioned $240 \mathrm{~mm}$ behind the sample to record the number of transmitted protons. This detector was aligned with the incident beam by maximizing the count rate as the detector was translated over the two perpendicular directions to the incident beam with no sample in the beam path. The detector was on a stage that was translated in $1 \mathrm{~mm}$ steps over a $30 \mathrm{~mm}$ range with a accuracy of $0.2 \mathrm{~mm}$ and a precision of $0.2 \mathrm{~mm}$ and alignment was accurate to within $\sim 0.2 \mathrm{~mm}$. Moving this detector in $1 \mathrm{~mm}$ increments allowed transmission data to be collected in approximately 0.1 degree angular increments to a maximum of $\sim 4$ degrees from the incident beam direction.

The collimated proton beam spot size was $1 \mathrm{~mm} \times 1 \mathrm{~mm}$ and the beam current was typically a few thousand ions per second. This count rate was primarily achieved by defocusing the beam prior to it being incident on a pair of slits approximately 8 meters upstream of the scattering chamber. The FWHM (full width at half maximum) angular profile of the incident beam was determined to be $0.25^{\circ}$. For data collection, the detector was typically translated in $1 \mathrm{~mm}$ steps over the $35 \mathrm{~mm}$ range through the peak maximum. Normalization of the transmission ion data for variations incident beam current was achieved with a second particle detector that remained fixed at a scattering angle of $30^{\circ}$ and subtending a solid angle of $\sim 0.1 \mathrm{sr}$ to the sample. For a given sample at a given beam energy the second detector recorded a 
preset number of scattered ions (typically 10,000) for the normalization. Data were collected for incident proton beam energies of 2.5-, 3.0-, and 3.5$\mathrm{MeV}$ on each sample. The end result was a plot of normalized ion flux versus angle for each sample and each beam energy.

\section{Ion Transport Modeling and Validation}

The Monte Carlo ion transport code TRIM (TRansport of Ions in Matter) was used to model the lateral straggling of $\mathrm{MeV}$-proton beams in metal foil targets. ${ }^{10,11}$ TRIM was originally developed to compute range and damage distributions of ions in amorphous solids and has since been used to study ion implantation, radiation damage, sputtering, and the reflection and transmission of ions. The code is generally applicable to ion energies ranging from about $0.1 \mathrm{keV}$ to several $\mathrm{MeV}$, depending on the masses involved. The lower ion energy limit results from using binary collision encounters only, while the upper limit is due to not including relativistic effects. Nuclear and electronic (the dominant mechanism in proton imaging) energy losses are treated as independent processes. In TRIM, ions lose energy due to nuclear events in discrete amounts while for electronic interactions the energy is lost continuously. The nuclear scattering also plays an important role in determining the deflection angles of the particle trajectories. A rigorous calculation of the scattering would require numerically evaluating the classical scattering integral for realistic interatomic potentials. This approach quickly becomes computationally intensive in Monte Carlo simulations, so TRIM uses an approximate analytical expression that has been shown to be valid to about $1 \%$ accuracy.

The TRIM ion transport model was used to determine the beam broadening distributions for $2.5-$ and $3.5-\mathrm{MeV}$ protons impinging on metal foil targets. Figures 1 to 6 show comparisons of the predicted and measured distributions. Also shown in the figures are the measured distributions for 3.0-MeV proton beams on each foil. Each TRIM simulation followed $10^{6}$ proton histories and took on the order of $1.5 \mathrm{hr}$ to complete using a $2.4 \mathrm{GHz}$ Intel $^{\circledR}$ Pentium PC. As seen in the figures, there is good qualitative agreement in the shapes of the calculated and experimental distributions but, generally, the predicted distributions tend to fall off more quickly than the experimental ones. The fall-off appears to be more prominent for the 2.5$\mathrm{MeV}$ beam energy cases, i.e., when greater energy loss occurs within the sample. The obvious exception is seen in Figure 6 for the gold foil. In this 
case, it is unknown why the opposite trend occurs, i.e., the experimental distributions fall off more rapidly than the predicted ones.

While the computed distributions are useful for validating the scattering models, they are less practical for incorporating into a beam spatial broadening correction algorithm. For the correction algorithm, it is preferable to characterize the broadening in terms of a simple parameterized value or expression. One possible parameter is the HWHM (half width at half maximum) of the distribution. Table 1 gives the HWHM values for the measured and predicted lateral straggling distributions for the cases of 2.5and $3.5-\mathrm{MeV}$ proton beams. The values were found by fitting high $(\geq 4)$ order polynomials to the discrete distributions shown in Figures 1 to 6 . The polynomial equations were then numerically solved to obtain the HWHM angles. As seen in the table, the measured and predicted values agree to within $20 \%$ over most of the range of materials and incident proton energies considered (also see Figure 7). This level of accuracy is adequate for the NIF target proton imaging characterization and modeling requirements.

Table 1. HWHM (Half Width at Half Maximum) values of the measured and predicted lateral straggling distributions from thin metal foils.

\begin{tabular}{|c|c|c|c|c|c|c|}
\hline \multirow[b]{2}{*}{ Foil } & \multirow[b]{2}{*}{$\begin{array}{l}\text { Nominal } \\
\text { Thickness } \\
\text { (microns) }\end{array}$} & \multirow[b]{2}{*}{$\begin{array}{l}\text { Gravimetric } \\
\text { Thckness } \\
\text { (microns) }\end{array}$} & \multicolumn{2}{|c|}{ 2.5-MeV Protons } & \multicolumn{2}{|c|}{ 3.5-MeV Protons } \\
\hline & & & $\begin{array}{c}\text { Measured } \\
\text { HWHM } \\
\text { (degrees) }\end{array}$ & $\begin{array}{c}\text { Predicted } \\
\text { HWHM } \\
\text { (degrees) }\end{array}$ & $\begin{array}{c}\text { Measured } \\
\text { HWHM } \\
\text { (degrees) }\end{array}$ & $\begin{array}{c}\text { Predicted } \\
\text { HWHM } \\
\text { (degrees) }\end{array}$ \\
\hline $\mathrm{Al}$ & 0.9 & 0.8967 & 0.391 & 0.288 & 0.254 & 0.227 \\
\hline $\mathrm{Ti}$ & 1.0 & 1.1270 & 0.655 & 0.504 & 0.431 & 0.344 \\
\hline $\mathrm{Ni}$ & 0.5 & 0.9890 & 0.949 & 0.805 & 0.542 & 0.545 \\
\hline $\mathrm{Ag}$ & 1.0 & 0.9367 & 1.082 & 0.949 & 0.634 & 0.681 \\
\hline W & 3.0 & 3.0077 & 3.564 & 2.945 & 2.391 & 2.012 \\
\hline $\mathrm{Au}$ & 1.0 & 1.1559 & 1.682 & 1.743 & 1.004 & 1.348 \\
\hline
\end{tabular}

Another possible parameterization of the beam broadening is the RMS (rootmean-square) scattering angle, $\theta_{R M S}$, for which an analytical expression has been previously derived by Wylie et al. ${ }^{5}$ Wylie's expression is based on combining Fermi's non-relativistic equation for the differential scattering angle and Bethe's equation for the target stopping power. Assuming small angle scattering, one obtains 


$$
\theta_{R M S}^{2}=\frac{m}{M} Z\left\lfloor\ln \left(\frac{\alpha}{\beta}\right) \ln \left(\frac{\ln \beta E_{\text {inc }}}{\ln \beta E_{\text {tran }}}\right)+\ln \left(\frac{E_{\text {inc }}}{E_{\text {tran }}}\right)\right\rfloor
$$

where $\alpha=a_{d} /\left(Z^{4 / 3} z e^{2}\right), \beta=4 m /(M I), E_{\text {inc }}$ is the incident ion energy, $E_{\text {tran }}$ is the transmitted ion energy, $e$ is the electronic charge, $Z$ is target atomic number, $z$ is the incident ion atomic number, $m$ is the electronic mass, $M$ is the incident ion mass, $a_{o}$ is the first Bohr radius of hydrogen, and $I$ is the effective ionization potential of the target. In Table 2 are the $\theta_{R M S}$ values for both the analytic expression and the TRIM simulations, again for 2.5- and $3.5-\mathrm{MeV}$ protons on the metal foils. The tabular $\theta_{R M S}$ values for the measured and TRIM distributions were computed by

$$
\theta_{R M S}^{2}=\sum_{n} \theta_{n}^{2}
$$

over the measured 0 to 3 degree interval, where $\theta$ is the scattering angle intensity of the $n^{\text {th }}$ angular bin in the discrete lateral straggling distribution. (Also note that the values of $E_{\text {tran }}$ used in the analytic expression were the computed averages of the transmitted proton energies from TRIM.) As seen in the table, the measured and TRIM RMS scattering angles are in very good agreement while analytic expression overestimates the broadening with increasingly greater error as the atomic number is increased.

Table 2. RMS (root-mean-square) scattering angles for $2.5-\mathrm{MeV}$ and 3.5$\mathrm{MeV}$ protons on thin metal foils.

\begin{tabular}{|c|c|c|c||c|c|c|}
\cline { 2 - 7 } \multicolumn{1}{c|}{} & \multicolumn{3}{c||}{$\theta_{\text {RMS }}($ degrees $)$} & \multicolumn{3}{c|}{$\theta_{\text {RMS }}$ (degrees) } \\
\hline Foil & Measured & TRIM & Wylie & Measured & TRIM & Wylie \\
\hline $\mathrm{Al}$ & 0.32 & 0.29 & 0.36 & 0.31 & 0.27 & 0.21 \\
\hline $\mathrm{Ti}$ & 0.41 & 0.36 & 0.88 & 0.37 & 0.31 & 0.50 \\
\hline $\mathrm{Ni}$ & 0.50 & 0.46 & 1.74 & 0.47 & 0.38 & 0.85 \\
\hline $\mathrm{Ag}$ & 0.53 & 0.49 & 1.76 & 0.44 & 0.42 & 0.97 \\
\hline $\mathrm{W}$ & 0.86 & 0.82 & 6.36 & 0.76 & 0.70 & 4.84 \\
\hline $\mathrm{Au}$ & 0.66 & 0.66 & 4.00 & 0.53 & 0.60 & 2.45 \\
\hline
\end{tabular}




\section{Conclusions}

The attainable resolution in transmission proton imaging requires a priori estimates of the degree of beam spatial broadening that occurs in the object being examined and a posteriori computational methods to mitigate its effect for improving the final image quality. A proton imaging correction algorithm has previously been demonstrated for partially correcting the effects of spatial broadening by accounting for the small angle multiple scattering of the ions as they traverse a sample. However, the algorithm does not explicitly treat objects comprised of compounds or mixtures, nor objects with complex material geometries. Imaging these types of objects will require voxel-dependent correction schemes that include scattering information derived from either direct material measurements or validated ion transport simulations. The objective of this study was to validate the lateral straggling distributions predicted by the TRIM Monte Carlo ion transport code. This was accomplished by comparing the computed distributions to recently measured transmitted proton distributions from thin calibration foils. For the proton energies and metal foil samples considered in this study, the TRIM-predicted lateral straggling distributions are found to be in good agreement with the measured distributions. 


\section{References}

1. A.E. Pontau, A.J. Antolak, D.H. Morse, A.A. Ver Berkmoes, J.M. Brase, D.W. Heikkinen, H.E. Martz, and I.D. Proctor, Nucl. Instrum. Meth. B40/41 (1989) 646.

2. A.E. Pontau, A.J. Antolak, D.H. Morse, and D.L. Weirup, Nucl. Instrum. Meth. B54 (1991) 383.

3. A.J. Antolak, G.S. Bench, A.E. Pontau, D.H. Morse, D.W. Heikkinen, and D.L. Weirup, Nucl. Instrum. Meth. A353 (1994) 568.

4. A.E. Pontau, A.J. Antolak, and D.H. Morse, Nucl. Instrum. Meth. B45 (1990) 503.

5. G.H. Bauer, A.J. Antolak, A.E. Pontau, D.H. Morse, D.W. Heikkinen, and I.D. Proctor, Nucl. Instrum. Meth. B43 (1989) 497.

6. A.J. Antolak, B.N. Handy, D.H. Morse, and A.E. Pontau, Nucl. Instrum. Meth. B59/60 (1991) 13.

7. A.J. Antolak and A.E. Pontau, Scanning Microscopy, Vol. 6, No. 1 (1992) 157.

8. G.S. Bench, A.J. Antolak, D.H. Morse, A.E. Pontau, A. Saint, G.J.F. Legge, Nucl. Instrum. Meth. B82 (1993) 447.

9. W.R. Wylie, R.M. Bahnsen, and H.W. Lefevre, Nucl. Instrum. Meth. 79 (1970) 245.

10. J.P. Biersack and L.G. Haggmark, Nucl. Instrum. Meth. 174 (1980) 257.

11. SRIM-2003, J.F. Ziegler and J.P. Biersack, http://www.srim.org. 


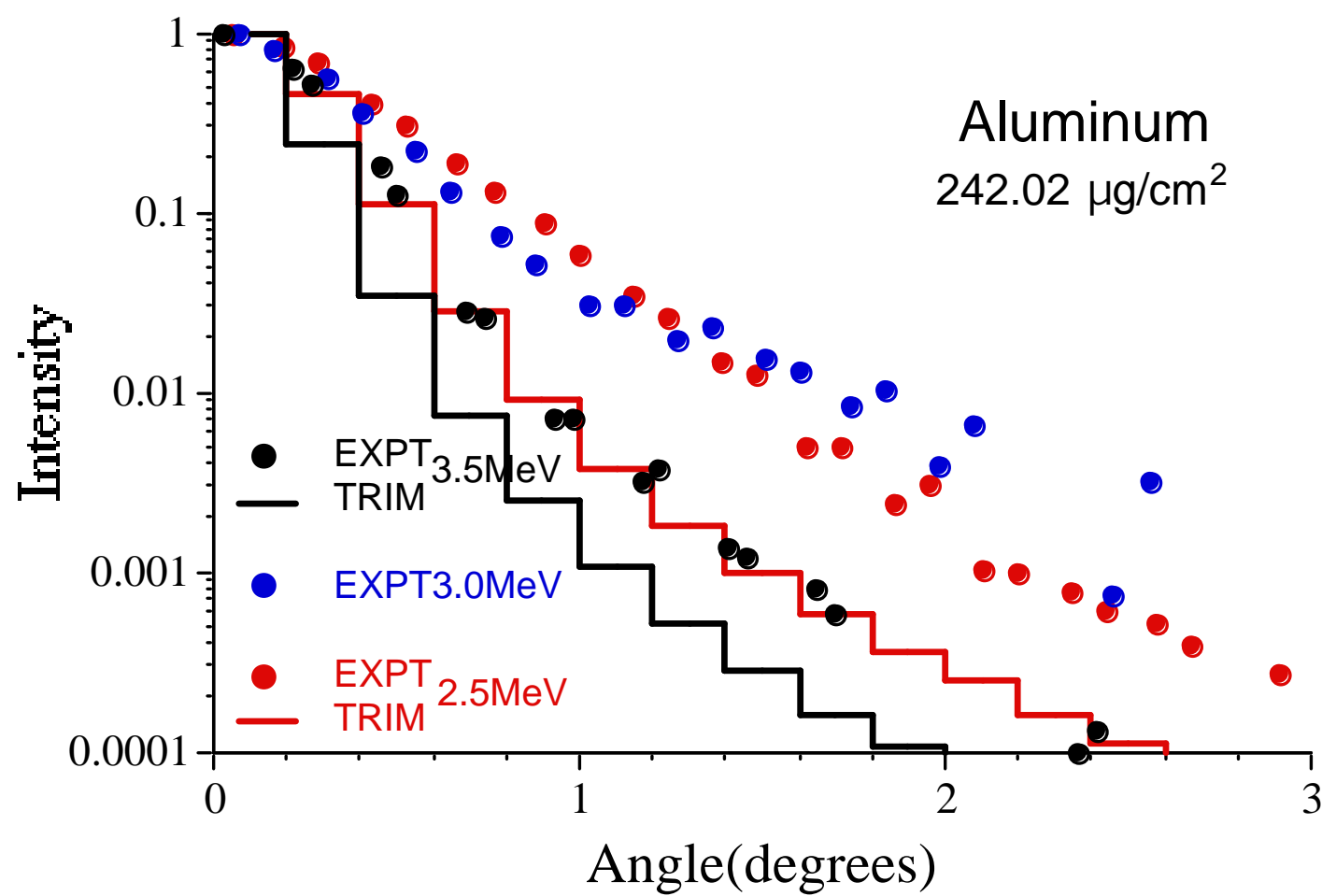

Figure 1. Comparison of the measured and calculated lateral straggling distributions for 2.5 - to $3.5-\mathrm{MeV}$ protons on a $0.8967 \mu \mathrm{m}\left(242.02 \mu \mathrm{g} / \mathrm{cm}^{2}\right)$ thick aluminum foil.

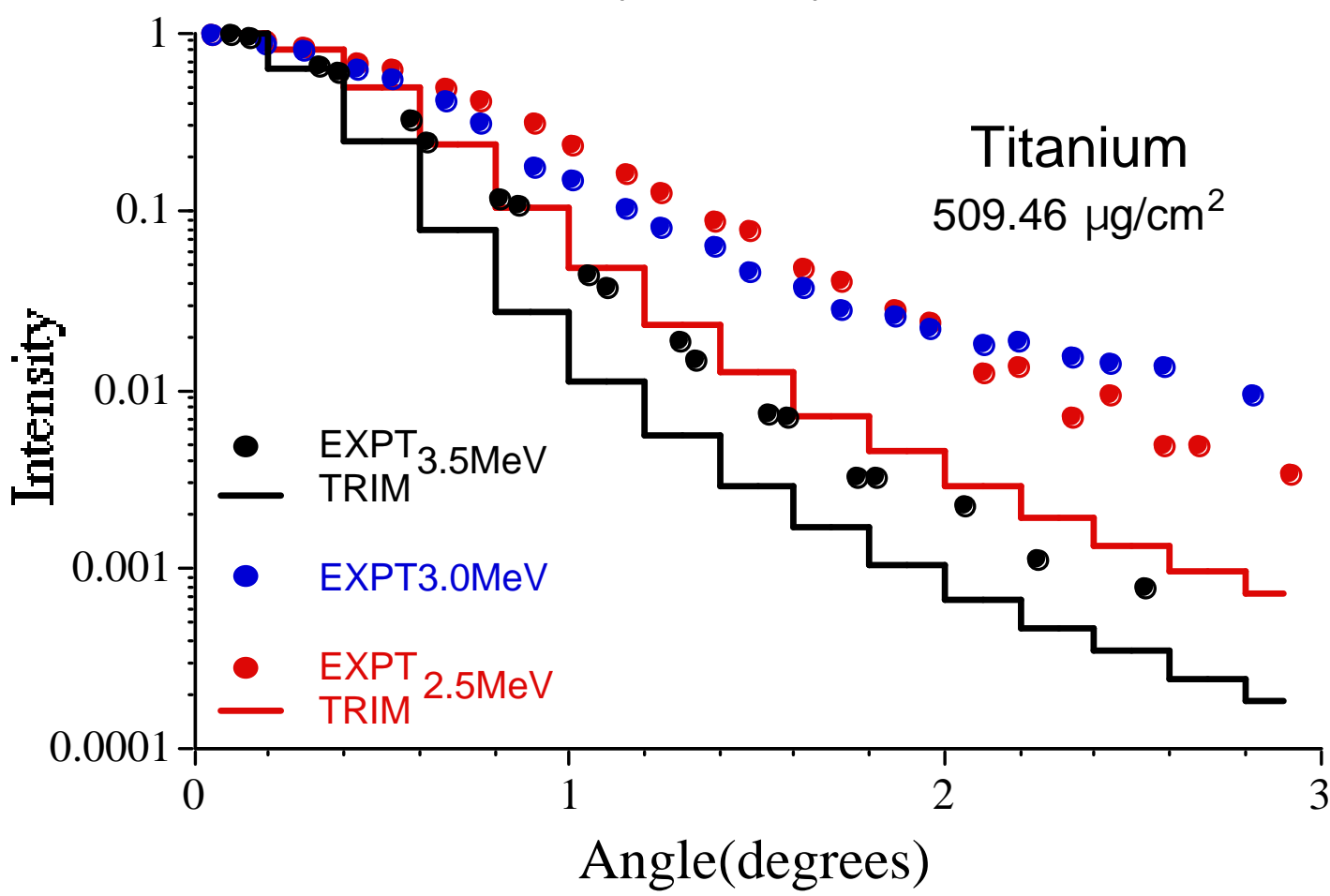

Figure 2. Comparison of the measured and calculated lateral straggling distributions for 2.5 - to $3.5-\mathrm{MeV}$ protons on a $1.127 \mu \mathrm{m}\left(509.46 \mu \mathrm{g} / \mathrm{cm}^{2}\right)$ thick titanium foil. 


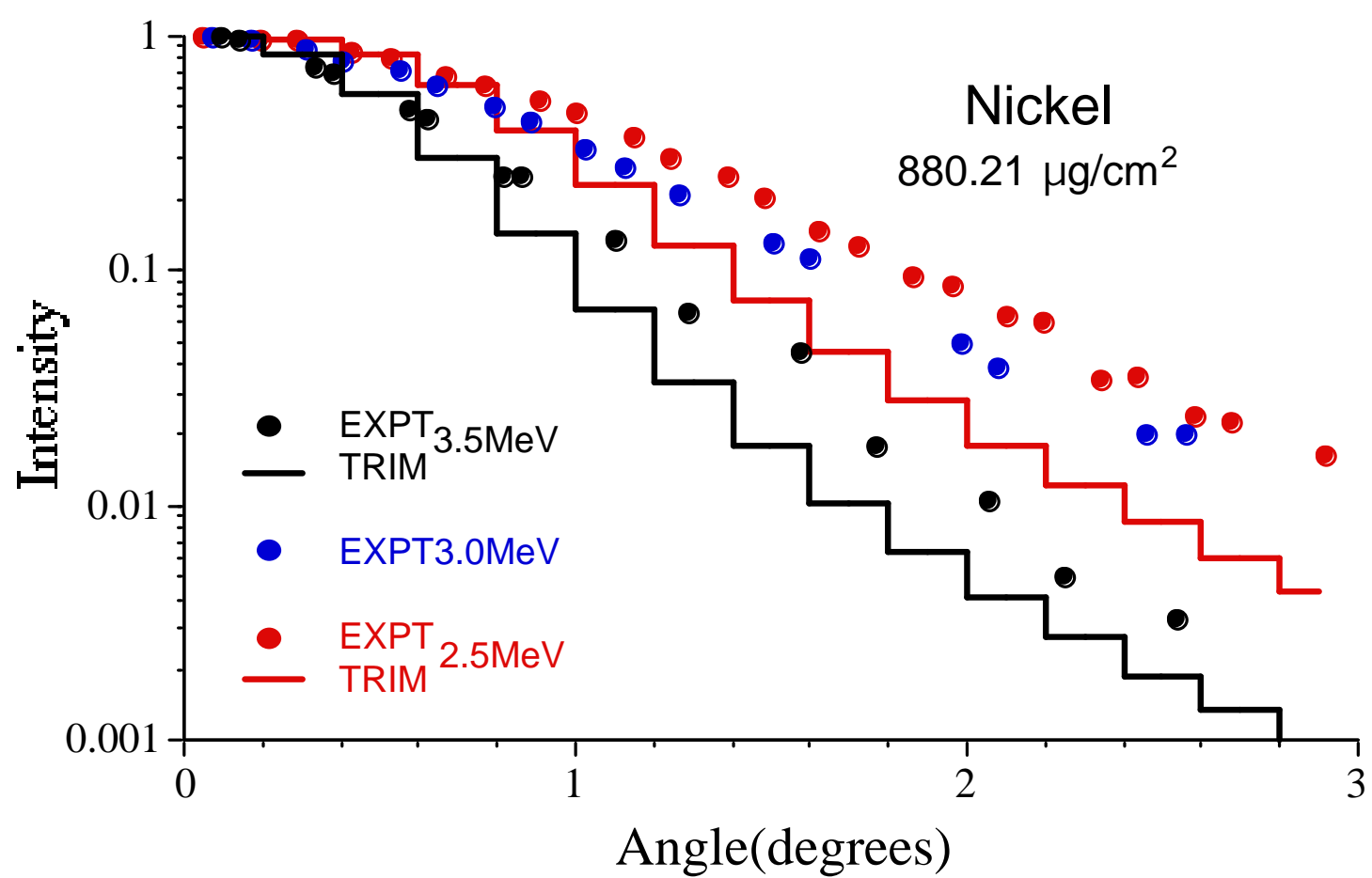

Figure 3. Comparison of the measured and calculated lateral straggling distributions for 2.5- to 3.5-MeV protons on a $0.9890 \mu \mathrm{m}\left(880.21 \mu \mathrm{g} / \mathrm{cm}^{2}\right)$ thick nickel foil.

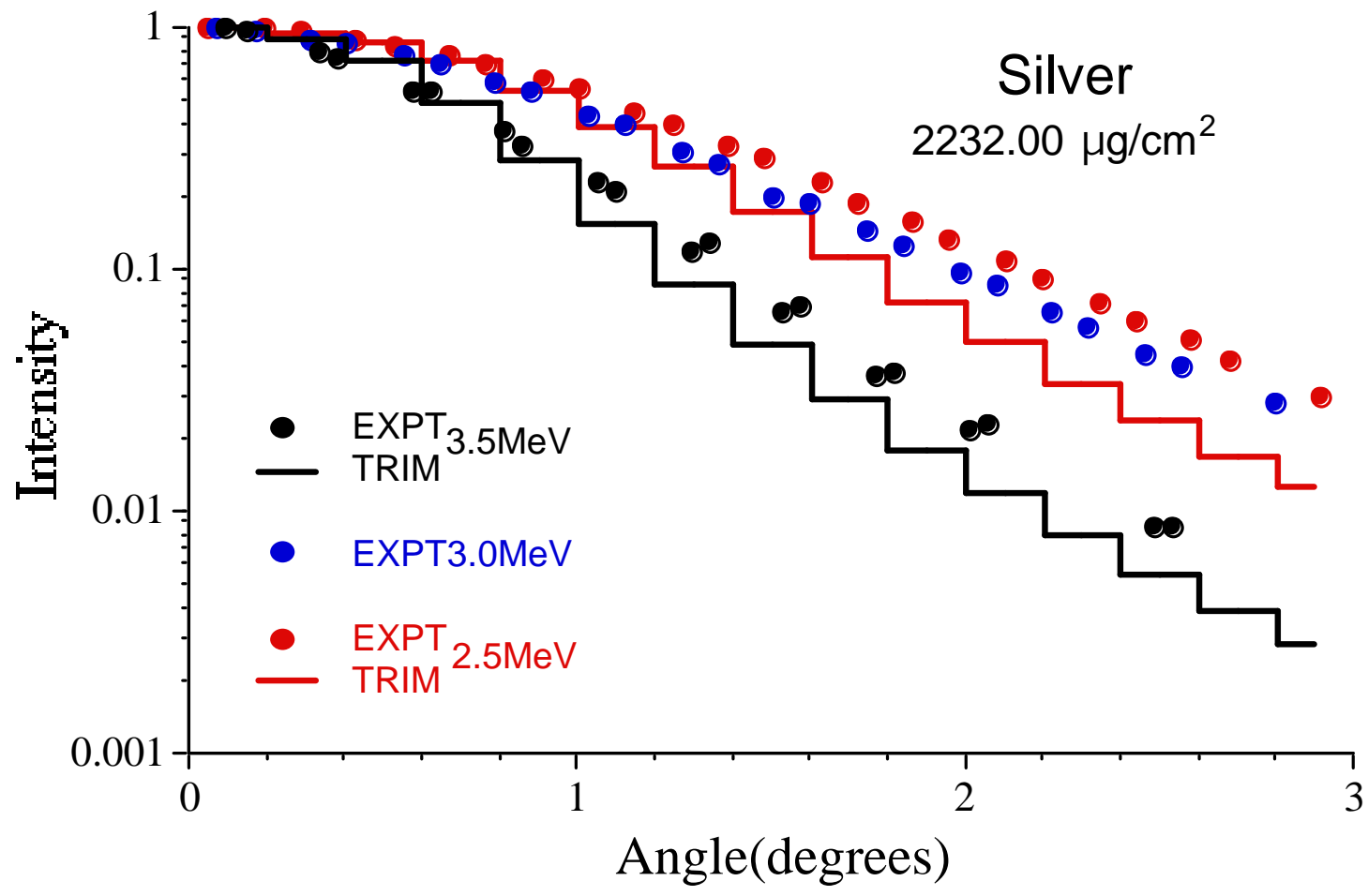

Figure 4. Comparison of the measured and calculated lateral straggling distributions for 2.5 - to $3.5-\mathrm{MeV}$ protons on a $0.9367 \mu \mathrm{m}\left(980.71 \mu \mathrm{g} / \mathrm{cm}^{2}\right)$ thick silver foil. 


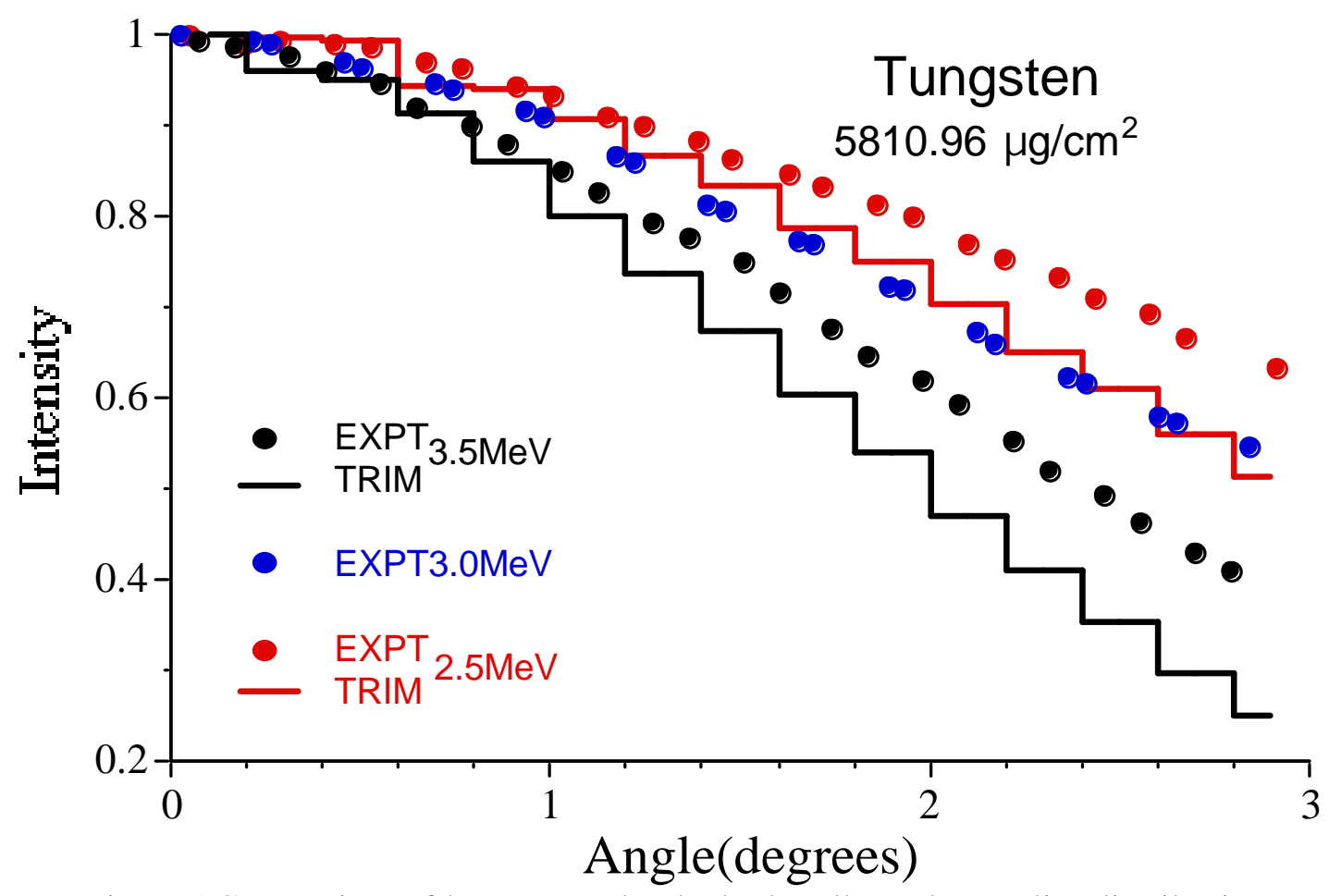

Figure 5. Comparison of the measured and calculated lateral straggling distributions for 2.5- to 3.5-MeV protons on a $3.0077 \mu \mathrm{m}\left(5810.96 \mu \mathrm{g} / \mathrm{cm}^{2}\right)$ thick tungsten foil.

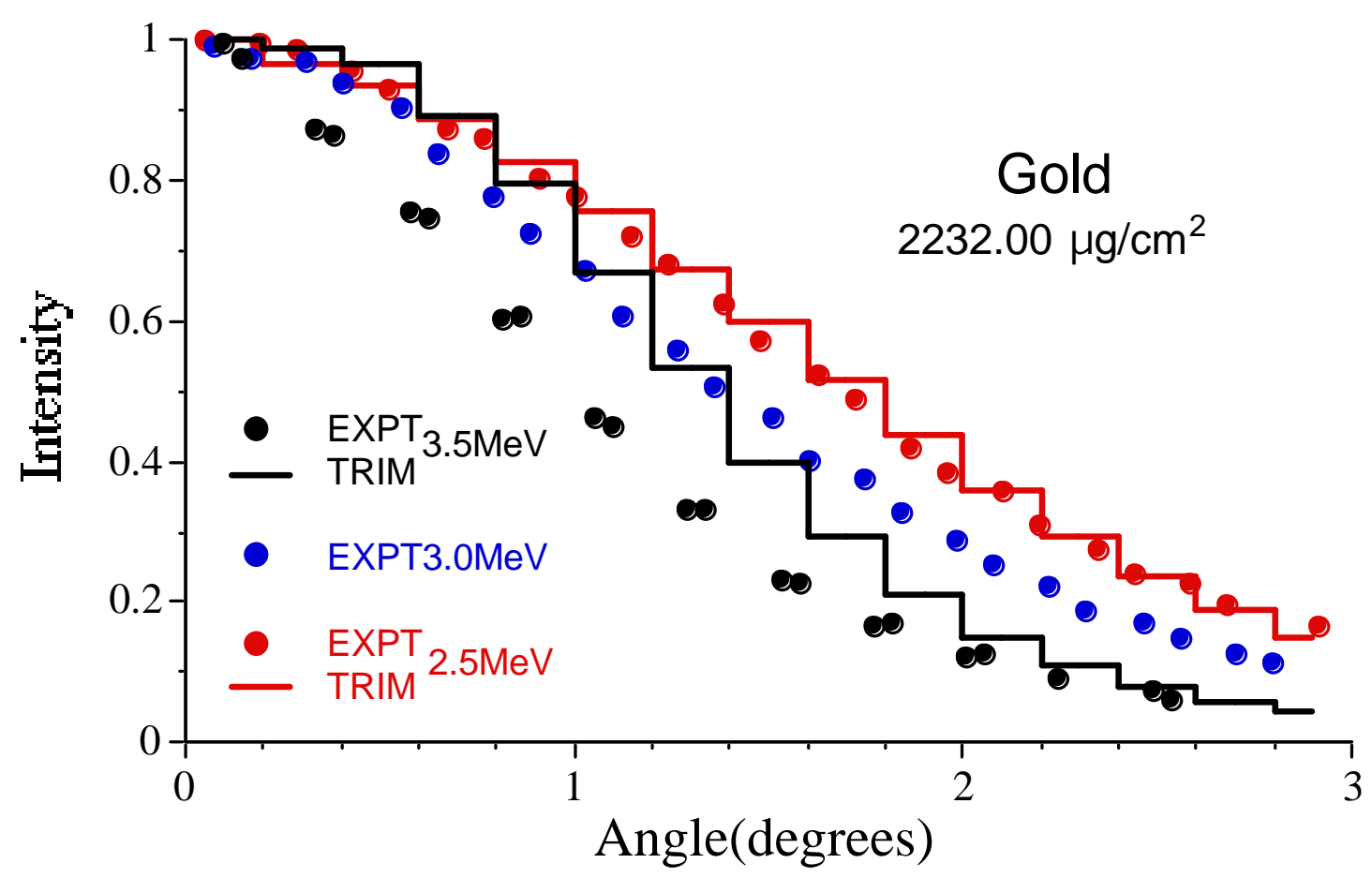

Figure 6. Comparison of the measured and calculated lateral straggling distributions for 2.5- to 3.5-MeV protons on a $1.1559 \mu \mathrm{m}\left(2232.00 \mu \mathrm{g} / \mathrm{cm}^{2}\right)$ thick gold foil. 


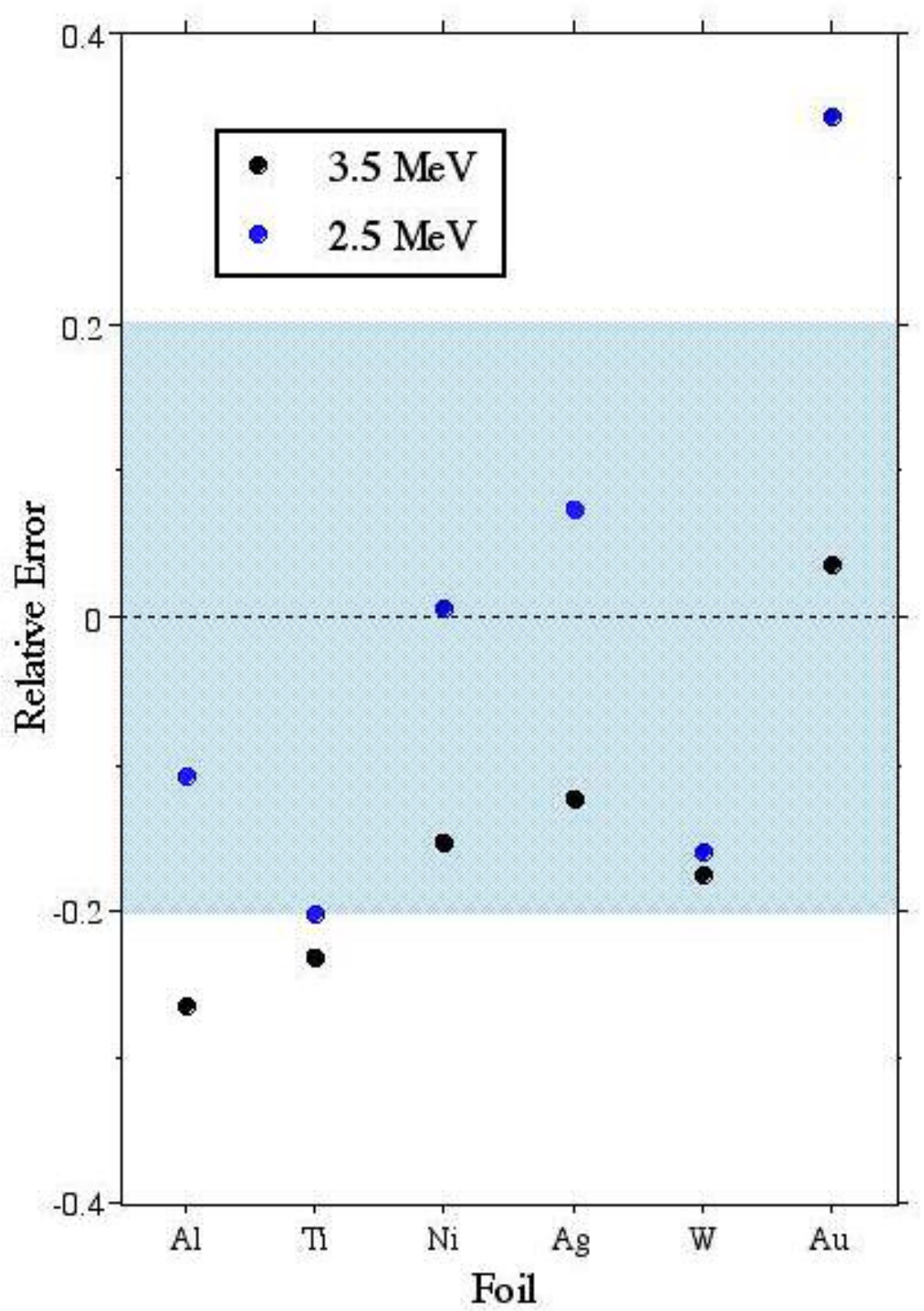

Figure 7. Relative error of TRIM-calculated lateral broadening HWHM values for $2.5-$ and $3.5-\mathrm{MeV}$ protons on thin metal foils. 


\section{Distribution}

4 M0830 Lawrence Livermore National Laboratory

Attn: Warren Hsing, L021

Harry Martz, L333

Thomas Felter, L356

Graham Bench, L397

PO Box 808

Livermore, CA 94550

$1 \quad$ MS 1056 Barney Doyle, 1111

1 MS 1196 Ray Leeper, 1677

1 MS 9402 Robert Bastasz, 8772

1 MS 9402 Rion Causey, 8772

5 MS 9403 Arlyn Antolak, 8773

1 MS 9403 Daniel Morse, 8773

1 MS 9403 Jim Wang, 8773

1 MS 9405 Ken Wilson, 8770

3 MS 9018 Central Technical Files, 8945-1

1 MS 0899 Technical Library, 9616

1 MS 9021 Classification Office, 8511/Technical Library, MS 0899, 9616

DOE/OSTI via URL 